\title{
Prefabricated Bar System for Immediate Loading in Edentulous Patients: A 5-Year Follow-Up Prospective Longitudinal Study
}

\author{
Enrico F. Gherlone, ${ }^{1}$ Gianpaolo Sannino $\left({ }^{1},{ }^{1}\right.$ Andrea Rapanelli, ${ }^{1}$ Roberto Crespi, \\ Giorgio Gastaldi, ${ }^{1,2}$ and Paolo Capparé ${ }^{1}$ \\ ${ }^{1}$ Department of Dentistry, IRCCS San Raffaele Hospital and Dental School, Vita Salute University, Via Olgettina, \\ No. 48, 20123 Milan, Italy \\ ${ }^{2}$ San Rocco Clinical Institute, Via dei Sabbioni, No. 24, Ome, 25050 Brescia, Italy \\ Correspondence should be addressed to Gianpaolo Sannino; gianpaolosannino@gmail.com
}

Received 27 September 2017; Accepted 31 January 2018; Published 27 February 2018

Academic Editor: Gasparini Giulio

Copyright (C) 2018 Enrico F. Gherlone et al. This is an open access article distributed under the Creative Commons Attribution License, which permits unrestricted use, distribution, and reproduction in any medium, provided the original work is properly cited.

\begin{abstract}
Objectives. The aim of this clinical study was to evaluate a new type of prefabricated bar system, supported by axial and tilted implants at 5-year follow-up. Materials and Methods. Twenty-nine consecutive participants (19 females, 10 males) (mean age 61.4 years), edentulous in one or both jaws, with severe atrophy of the posterior regions, were treated according to the All-on-four ${ }^{\circledR}$ protocol with immediately loaded axial (64) and tilted (64) implants supporting complete-arch screw-retained prostheses (12 maxillary, 20 mandibular) featuring a prefabricated bar as framework. Follow-up visits were performed at 3, 6, 12, 24, 48, and 60 months after implant insertion. Radiographic assessments were made using panoramic radiographs obtained immediately after surgery and at each follow-up visit. Bone level measurements around the axial and tilted implants were compared by means of the Student's $t$-test. Results. One axial implant failed in the lower jaw and did not compromise prosthetic function. The 60-month overall implant survival rate was $100 \%$ for axially positioned implants and $98.44 \%$ for tilted implants. The implant survival rates were $100 \%$ in the maxilla and $98.75 \%$ in the mandible. None of the 32 fixed prostheses were lost during the observation period, representing a prosthetic survival rate of $100 \%$. No statistically significant differences $(P>0.05)$ in marginal bone loss between tilted and axial implants were detected in either jaw over time. Conclusions. The use of the evaluated prefabricated bar for immediately loaded implants placed according to the All-on-four concept may significantly reduce implant failures; however, more long-term prospective clinical trials are needed to affirm the effectiveness of the surgical-prosthetic protocol.
\end{abstract}

\section{Introduction}

Clinical implant dentistry is oriented to low cost treatments using simple protocols that are well supported by scientific data, while providing immediate function through immediate restoration and loading of dental implants [1-4].

The All-on-four concept that employs tilted implants to restore edentulous patients has been proposed as an alternative to bone augmentation procedures [5].

The placement of four implants, two implants tilted posteriorly and two vertical implants in the anterior region, allows for avoiding bone augmentation procedures when rehabilitating a completely edentulous jaw with minimal bone volume [6]. The development to tilting of fewer implants has been encouraged by the results from implant load analyses, demonstrating that four implants are enough for complete-arch prosthesis $[7,8]$.

Longer implants may be optimally placed in areas with good cortical anchorage to increase prosthetic support and reduce the length of the cantilever. This procedure supported a simpler, less expensive, and less time-consuming treatment compared to maxillary sinus lift or bone grafts $[9,10]$.

Krekmanov et al. treated forty-seven consecutive patients with implants placed in tilted positions: the cumulative success rates in the maxilla at 5 years were $98 \%$ for tilted implants and 93\% for nontilted implants [11].

Analysis of the load distribution in one mandibular case showed no significant difference between tilted and the 
nontilted implants, and the improved prosthesis support was confirmed. The immediate loading of tilted implants with a provisional restoration has been proposed as a simpler, more predictable, less expensive, and less time-consuming treatment of the atrophic maxilla [12]. However a passive fit of the framework plays a key role in splinting and loading nonparallel implants. Tensile, compressive, and bending forces may be dangerous for the osseointegration process and/or result in failure of the components [13].

Soldering or laser welding procedures are often needed to compensate loss of accuracy due to clinical/laboratory errors and achieve the appropriate adaptation of the framework. Using prefabricated parts to assemble prosthetic frameworks, such as bars, could be useful in reducing material distortion, chair time, and the high costs of fabrication. Therefore the aim of this clinical study was to evaluate the use of a prefabricated bar system for immediately loaded implants in patients rehabilitated according to the All-on-four concept with up to 5 years' follow-up.

\section{Materials and Methods}

2.1. Patient Selection. This prospective longitudinal study was performed at the Department of Dentistry, San Raffaele Hospital, Milan, Italy. Between March 2011 and March 2012, 29 participants (19 women, 10 men), aged between 41 and 72 years (mean age 61.4) were consecutively treated according to the All-on-four protocol with immediately loaded axial (64) and tilted (64) implants supporting complete-arch screwretained prostheses (12 maxillary, 20 mandibular). Four patients were treated with both maxilla and mandibular prosthetic rehabilitation.

The investigation was conducted according to the tenets of the Helsinki Declaration and followed STROBE (Strengthening the Reporting of Observational Studies in Epidemiology) guidelines (https://www.strobe-statement.org). The study was approved by the appropriate ethics committees related to the institution in which it was performed and written informed consent was obtained from each participant. The following inclusion criteria were adopted: all patients were in good health, patients had to be edentulous (in one or both jaws) or they had to have a few hopeless teeth, severe atrophy of the mandible or maxilla in posterior regions. Exclusion criteria were the presence of any active infection or severe inflammation in the areas intended for implant placement, presence of chronic systemic disease, any interfering medication such as steroid therapy or bisphosphonate therapy, radiation therapy to head or neck region within 5 years, smoking more than 15 cigarettes, bruxism habits, and poor oral hygiene.

The diagnosis was made clinically and radiographically (preoperative panoramic radiograph and CT scan) (Figure 1). Study casts were obtained from jaw impressions of the patients and mounted on articulators. For edentulous patients, new removable prostheses were fabricated in order to restore optimal occlusal vertical dimension, mandibular position, and occlusal planes. The new prostheses were duplicated and used as radiographic templates during the

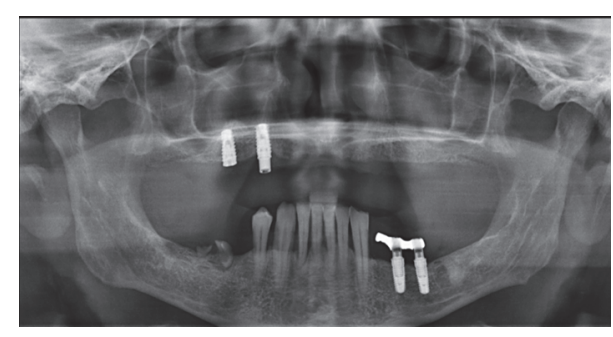

FIGURE 1: A preoperative panoramic radiograph.

TABLE 1: Implant diameters and lengths for maxilla and mandible ( maxilla $n=$ implant $=48$; mandible $n=$ implant $=80$ ).

\begin{tabular}{llcc}
\hline & & $\begin{array}{c}\text { Length } \\
13 \mathrm{~mm}\end{array}$ & Length $15 \mathrm{~mm}$ \\
\hline \multirow{2}{*}{$\begin{array}{l}\text { Mpright } \\
n=24\end{array}$} & Diameter $4.5 \mathrm{~mm}$ & 10 & 0 \\
Tilted & Diameter $3.8 \mathrm{~mm}$ & 14 & 0 \\
$n=24$ & Diameter $4.5 \mathrm{~mm}$ & 12 & 12 \\
\hline \multirow{3}{*}{ Upright $n=40$} & Diameter $3.8 \mathrm{~mm}$ & 0 & 0 \\
\multirow{2}{*}{ Tilted } & Mandible $n=80$ & \\
$n=40$ & Diameter $4.5 \mathrm{~mm}$ & 4 & 10 \\
& Diameter $3.8 \mathrm{~mm}$ & 8 & 18 \\
& Diameter $4.5 \mathrm{~mm}$ & 18 & 14 \\
& Diameter $3.8 \mathrm{~mm}$ & 4 & 4 \\
\hline
\end{tabular}

CT exams. The bone volume was accurately assessed for a safe and prosthetically driven implant placement. All patients gave their written informed consent for immediate implant loading.

2.2. Surgical Procedure. One hour prior to surgery the patients received $2 \mathrm{~g}$ amoxicillin (Zimox, Pfizer Italia, Latina, Italy) and $1 \mathrm{~g}$ twice a day for a week after surgical procedure. Surgery was performed under anesthesia induced by local infiltrations of optocain solution with adrenaline 1:80.000 (AstraZeneca, Milan, Italy).

In edentulous mandibles, incisions were made on top of the alveolar crest, from the first molar on one side to the first molar on the contralateral side with bilateral releasing incisions. Subperiosteal dissection on the lingual and vestibular surfaces was carried out and mental foramina were sited. The most posterior implants were placed close to the anterior wall of the mental loop and were tilted distally about 25-30 degrees relative to the occlusal plane. The posterior implants, which were $4.5 \mathrm{~mm}$ in diameter and 15 or $13 \mathrm{~mm}$ in length, typically emerged at the second premolar position. Anterior implants were either 4.5 or $3.8 \mathrm{~mm}$ in diameter and $13 \mathrm{~mm}$ in length (Winsix, Biosafin, Ancona, Italy) (Table 1). After placement of the posterior implants bilaterally, additional implants were placed in the anterior space.

In some cases tooth extractions were carried out and, when necessary, bone shaping was performed with a round 


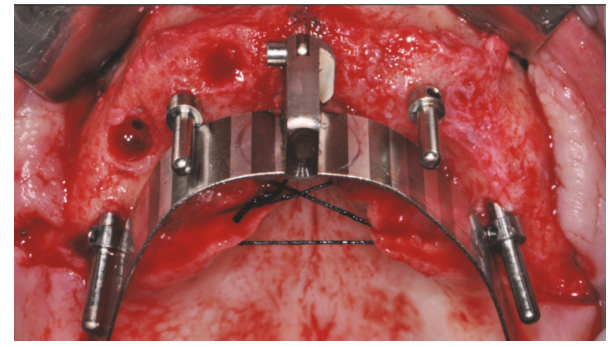

FIGURE 2: Implant site preparations assisted by a customized surgical guide.

bur to level the bone crest and, to achieve crestal positioning, bone recontouring was performed distal to the angled implants.

In edentulous maxillary patients, incisions were made on the alveolar crest from the first molar on one side to the first molar on the contralateral side with bilateral releasing incisions. Subperiosteal dissection was carried out. The most posterior implant was placed close to and parallel with the anterior sinus wall. Thus, this implant was tilted distally approximately 25 to 30 degrees. The lower corner of the implant neck was positioned at bone level (Figure 2).

Then the placement of implants in the anterior part of the maxilla was performed, and the implant neck was positioned at bone level. The posterior implants were $4.5 \mathrm{~mm}$ in diameter and 15 or $13 \mathrm{~mm}$ in length, and the anterior implants were either 4.5 or $3.8 \mathrm{~mm}$ in diameter and $13 \mathrm{~mm}$ in length (Winsix, Biosafin, Ancona, Italy) (Table 1).

Underpreparation was performed in soft bone to obtain high primary stability. The implant in immediate function had a final insertion torque ranging between 30 and $40 \mathrm{~N} / \mathrm{cm}$. Angulated abutments (Extreme Abutment, EA ${ }^{\circledR}$ Winsix, Biosafin, Ancona, Italy) for anterior implants were set at $17^{\circ}$ and those for posterior implants at $30^{\circ}$ to compensate for the lack of parallelism between implants as well as to place the prosthetic screw-access holes in an occlusal or lingual location. The angulated abutments were tightened with $25 \mathrm{~N} / \mathrm{cm}$ of torque. Flap adaptation and suturing were performed in the usual manner with 4-0 nonresorbable suture (Vicryl; Ethicon, Johnson \& Johnson, New Brunswick, NJ, USA).

Nonsteroidal anti-inflammatory drugs (Brufen $600 \mathrm{mg}$, Abbott Laboratories, Chicago, IL, USA) and chlorhexidine digluconate $0.2 \%$ mouthwash during the first 2 weeks after surgery were prescribed as postoperative care for all participants.

2.3. Prosthetic Protocol. After surgery straight cylinders (AT, WINSIX; BiosafIn) were screwed onto the angulated abutment. The interimplant distance was measured (Figure 3(a)). Three bar tubes were shortened to the optimal lengths using the specific cutter bar device and a splitter disk [14]. Two bar joints (CF and CM, WINSIX; BiosafIn) were inserted into the end of each tube in order to connect the whole structure to the cylinders (Figure 3(b)). The height of the cylinders was chosen to make the bar parallel to the occlusal plane. All the joints were connected to the cylinders and fixed by means of resin cements. No soldering was performed; the universal nature of the ball joint allows the tube bar to be located in the horizontal plane in a truly stress free alignment [15]. The prostheses were provided with four large openings according to planned cylinder emergence. The passive seating and the occlusal relationship of the removable prostheses were checked (Figure 4 ). The bar system was attached to the denture with self-curing acrylic resin.

After polymerization, the prostheses with the incorporated bar system were removed from the implants and retention, marginal precision, and stability were improved by resin addition around the collar of the cylinders (Figures 5(a) and 5(b)). Screw-retained full-arch temporary prostheses were placed (Figure 6).

Cantilevers were extended to the first molar regions and in three cases only to second premolar. Articulating paper (Bausch Articulating Paper, Nashua, NH, USA) was used to check the occlusion and adjust it, if necessary. Static occlusion consisted of central contacts established on all masticatory units. Dynamic occlusion included canine/premolar guidance, regardless of the opposite arch settings. Screw-access holes were covered with provisional resin (Fermit, Ivoclar Vivadent, Naturno, Bolzano, Italy). Fifteen days after prosthesis delivery, a final occlusal adjustment was performed.

All patients followed a soft/liquid diet for 2 months (the bread consistency varied).

After 4 months from implant positioning, 32 definitive prostheses were placed (Figure 7).

2.4. Follow-Up. Follow-up visits were performed by a dental hygienist, trained for clinical studies, and calibrated at 3 , $6,12,24,36,48$, and 60 months after implant insertion. Success criteria for implant survival were presence of implant stability, absence of radiolucent zone around the implants, no mucosal suppuration, and no pain. Restoration success was defined as the absence of fractures of the acrylic resin superstructure. Implant survival was defined as the absence of implant mobility, swelling, or pain in the surgical site at the time of examination.

Implant success was defined as implant survival plus marginal bone loss of less than $1.5 \mathrm{~mm}$ after 1 year of loading and no more than $0.2 \mathrm{~mm}$ of loss between each follow-up appointment after the first year of function.

2.5. Radiographic Examination. Radiographic assessments were made using panoramic radiographs obtained immediately after surgery and at each follow-up visit (Figure 8 ). Bone level measurements were performed on the mesial and distal aspect of each implant, using the implant-abutment junction as a reference point.

To adjust for dimensional distortion and enlargement on the radiographs, the actual sizes of the implants were compared to the measured implant dimensions on the radiograph $[1,15]$. A radiologist twice measured the changes in marginal bone height over time: the reference points were marked and the lines were measured on the screen interactively (the numeric value of measurements was reported by software) (CDR, Schick Technologies, Long Island City, NY, USA). The 


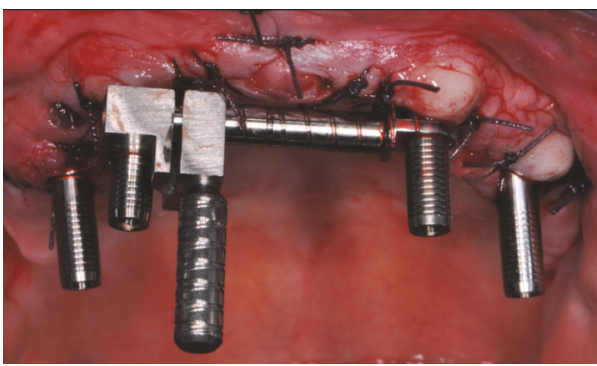

(a)

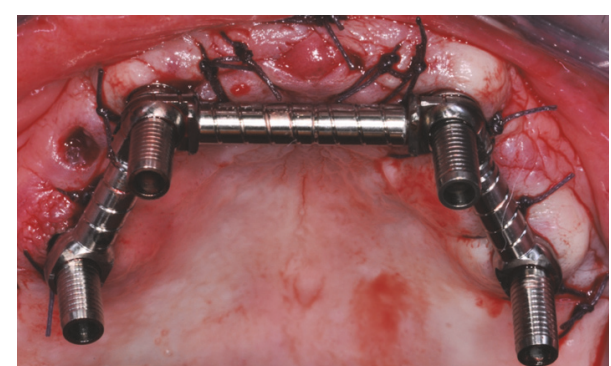

(b)

FIGURE 3: Each tube was shortened at the correct implant distance (a) and connected to adapters by using two dedicated bar joints in order to develop the whole bar structure (b).

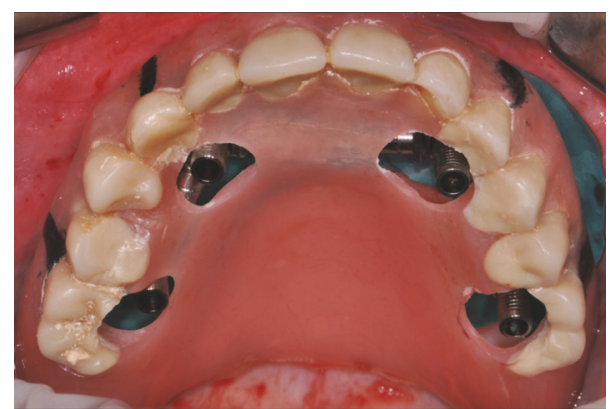

FIGURE 4: Passive seating and occlusal relationship of the removable prostheses were checked. Removable prostheses were released in correspondence with the expected bar volume. The contacts between the prostheses and the mucosal regions, which were not involved in the surgical procedures, were used as anatomical preand postsurgical landmarks to avoid variations in the occlusal relationships previously achieved. Four large openings were made according to planned cylinder emergence.

implant length (a known dimension) was used for calibration. The radiographic measurements were compared to the values obtained immediately after surgery.

2.6. Statistical Analysis. A dedicated software (SPSS 11.5.0, SPSS, Chicago, IL, USA) was used for all statistical analyses. Sample size calculation was performed before patients recruitment. Bone level measurements were reported as means \pm standard deviations at 6,12 , and 60 months. Bone loss around the upright and tilted implants was compared by means of the Student's $t$-test at a significance level of $P=0.05$.

\section{Results}

In the first four months after implant placement, one implant failed (one mandibular), as a result of painfulness (Table 2). The failed implant was axial and did not compromise prosthetic function. An implant of the same length and larger diameter was immediately placed and left unloaded until a new definitive prosthesis was completed and inserted.

The 5-year overall implant survival rate was $100 \%$ for axially positioned implants and $98.44 \%$ for tilted implants.
TABLE 2: Failure table for upright and tilted implants (maxilla $n=$ implant $=48$; mandible $n=$ implant $=80$ ).

\begin{tabular}{lccc}
\hline & Placed & Failed & Survival (\%) \\
\hline Upright & & Maxilla & $n=48$ \\
Tilted & 24 & 0 & 100 \\
\hline & 24 & 0 & 100 \\
Upright & 40 & Mandible $n=80$ & \\
Tilted & 40 & 1 & 97.50 \\
\hline
\end{tabular}

The implant survival rates were $100 \%$ in the maxilla and $98.75 \%$ in the mandible.

None of the 32 fixed prostheses were lost during the observation period, representing a prosthetic survival rate of $100 \%$. Occlusal screw loosening, was observed in $3.03 \%$ of cases ( 4 implants) within 6 months of follow-up.

Radiographic results are reported in Table 3. At the 60month evaluation, peri-implant crestal bone loss averaged $1.08 \pm 0.45 \mathrm{~mm}$ for upright maxillary implants $(n=24$ implants) and $1.02 \pm 0.67 \mathrm{~mm}$ for tilted maxillary implants ( $n=24$ implants) (Table 3 ). In the mandible, a mean peri-implant crestal bone loss of $1.04 \pm 0.61 \mathrm{~mm}$ for upright implants $(n=40)$ and $1.09 \pm 0.56 \mathrm{~mm}$ for tilted implants $(n=40)$ was found (Table 3$)$.

No statistically significant differences $(P>0.05)$ in crestal bone loss between tilted and upright implants were detected at 6-, 12-, and 60-month follow-up evaluation in either jaw.

\section{Discussion}

The data from the present prospective study have shown encouraging clinical results as a means of restoring edentulous jaws with immediately loaded full-arch fixed prostheses supported by a prefabricated bar system and screwed onto two anterior axial implants and two distal tilted implants.

One loaded implant was lost, and the prosthesis survived on the remaining three implants until the replacement implant was loaded. The use of three loaded implants allows for the failure of one implant without failure of the prosthesis. The failed implant was inserted with a torque of at least $40 \mathrm{Ncm}$. 


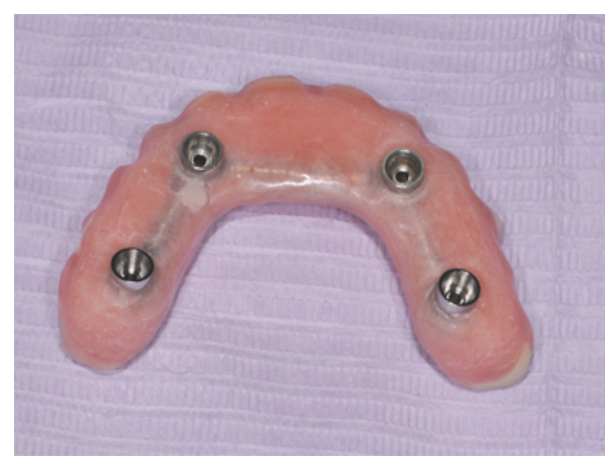

(a)

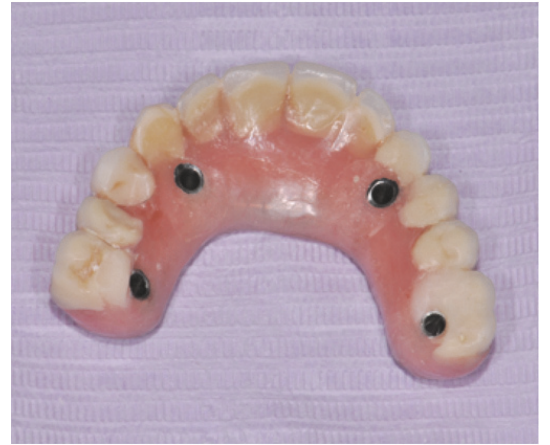

(b)

FiguRE 5: The bar was attached to the denture with self-curing acrylic resin and marginal precision was improved by resin addition around the collar of the adapters ((a) crestal view, (b) occlusal view).

TABLE 3: Crestal bone loss values (mean \pm SD) for maxillary and mandibular tilted and upright implants (maxilla $n=$ implant $=48$; mandible $n=$ implant $=80$ ).

\begin{tabular}{lcccc}
\hline Bone Loss & \multicolumn{2}{c}{ Upright } & & Tilted \\
& Maxilla $n=24$ & Mandible $n=40$ & Maxilla $n=24$ & $1.00 \pm 0.32$ \\
\hline 6 months $(\mathrm{mm})$ & $0.99 \pm 0.23$ & $0.97 \pm 0.31$ & $1.06 \pm 0.50$ & $1.01 \pm 0.27$ \\
12 months $(\mathrm{mm})$ & $1.03 \pm 0.33$ & $1.05 \pm 0.44$ & $1.02 \pm 0.67$ & $1.08 \pm 0.41$ \\
60 months $(\mathrm{mm})$ & $1.08 \pm 0.45$ & $1.04 \pm 0.61$ & $1.09 \pm 0.56$ \\
\hline
\end{tabular}

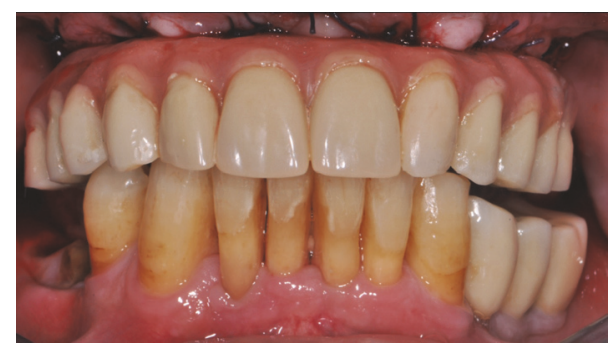

Figure 6: Provisional screw-retained bar-reinforced acrylic resin prosthesis delivery. Frontal view.

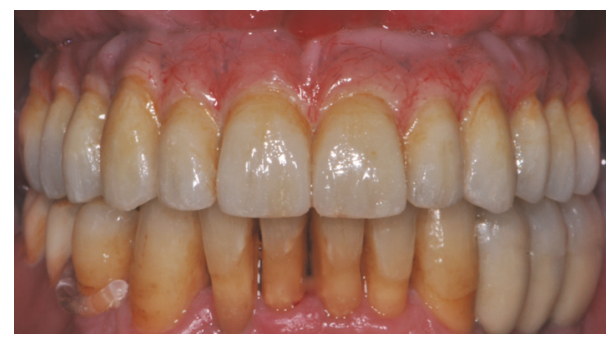

Figure 7: Definitive screw-retained full-arch restoration at 60month follow-up.

In the current study, radiographs demonstrated that the bone resorption pattern for posterior, angulated implants was similar on the mesial and distal surfaces. This was in agreement with the findings of other studies [8-10, 16-18].

No statistically significant differences $(P>0.05)$ in crestal bone loss between tilted and upright implants were

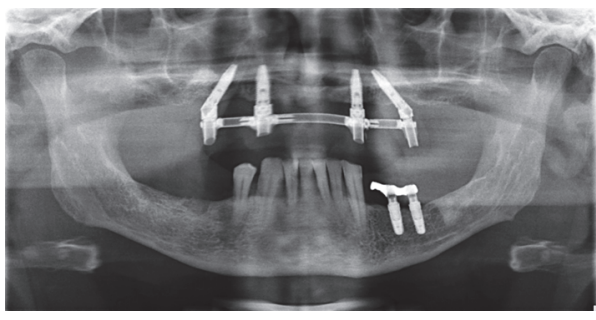

FIGURE 8: Panoramic radiograph at 60-month follow-up.

detected at 6- and 12-month follow-up evaluation in either jaw, and this is also consistent with other data found in the literature, confirming that tilted implants may achieve the same outcome as implants placed in an upright position $[6,16]$. This positive result is associated with biomechanical advantages, since in this protocol implants are placed in strategic positions from a load-sharing point of view. Placement of the 2 well-anchored posterior tilted implants together with the anterior upright implants can provide a predictable foundation for an implant-supported prosthesis. This surgical-prosthetic procedure also seems to validate the reduced length of the prostheses cantilevered segments. Implant placement and orientation provided effective crossarch stabilization without the need for bone grafting procedures. Excluding maxillary sinus bone grafts resulted in significantly less morbidity and dramatically decreased the financial costs associated with those procedures.

This treatment protocol allows the implant rehabilitation to be simplified and shortened for both the patient and the clinical team. The postsurgical period is more comfortable for 
patients since they have been utilizing their fixed prosthesis from the first day [9].

The immediate creation of the temporary restoration with a simple and repeatable prosthetic protocol represents a major advantage for patients, providing less expensive and less time-consuming treatments [10].

Traditional laboratory procedures such as soldering and welding may give rise to errors and increase in cost; furthermore, several bar framework material, such as gold alloy, silver-palladium alloy, commercially pure titanium, and cobalt-chromium alloy transfer significant stress to the supporting peri-implant tissues [19].

The key of prefabricated precision-milled components is that the framework is assembled without the use of soldering, laser welding, or conventional bonding techniques, thus reducing stress transmission to bone around the implants.

There is no casting, soldering, laser welding, or bonding of components when fabricating the definitive bar. This, combined with the universal ball joint nature of the components, ensures a true passive fit when the bar is assembled and seated.

No laboratory time is required to fabricate the bar and there are no gold-alloy charges. Clinically, there is no need for the bar sections to be soldered in an attempt to achieve passive fit-a step that may need repeating-as with the conventional method. The prefabricated bar is relatively inexpensive compared with conventional gold castings and CAD/CAM options.

Precision-milled components provide an improved quality of fit. The physical and mechanical properties of the component materials can be controlled accurately, which is difficult to achieve with conventional casting methods [14]. The passive-fit bar assembly can result in greatly reduced stress transmission to the supporting implants [14]. Studies have demonstrated that this is also a viable treatment option for immediate-loading situations in the mandible, provided that the implants achieved insertion torques exceeding $50 \mathrm{Ncm}$ approximately [20].

A clinical study [21] evaluated initial, 4-month, and 1year stability of immediately loaded dental implants inserted according to a protocol of lower rehabilitation with prefabricated bars. Immediately after implant installation, resonance frequency analysis (RFA) for each implant was registered as well as after 4 months and 1 year with the prosthetic bar removed as it is a screwed system. The analysis of variance showed a statistically significant result $(P=0.015)$ among implant stability quotient values for the different periods evaluated. Tukey test results showed statistically significant differences between 1-year results and the initial periods but there was no statistically significant difference between initial and 4-month results $(P>0.05)$.

Prefabricated bars were compared to custom-made bars used for implant-retained mandibular complete overdentures [22]. All patients were evaluated clinically and radiographically immediately after overdenture delivery and after 6,12 , and 18 months.

There was more pronounced bone resorption in cast bar group more than the prefabricated bar group and minimal marginal bone loss in the group treated with prefabricated bar. The prefabricated bar overdentures showed less bone resorption distal to the implants in comparison with the cast bar implant-retained overdentures. The prefabricated bar implant-retained overdenture showed low significant reduction in the bone height after 1 year, and a very highly significant reduction after 18 months.

\section{Conclusions}

Data and results of this clinical study demonstrated high success rates and a low number of complications.

The use of the evaluated prefabricated bar for immediately loaded implants placed according to the All-on-four concept may significantly reduce implant failures; however, more long-term prospective clinical trials are needed to affirm the effectiveness of the surgical-prosthetic protocol especially performed in various clinical centers from different clinicians.

\section{Ethical Approval}

Italian Ministry of Health approved the study, Protocol no. RF-FSR-2007-646412, Italian National Registry Office of Research, no. L1660026.

\section{Conflicts of Interest}

All the authors declare that there are no conflicts of interest regarding the publication of this paper.

\section{References}

[1] M. Hinze, T. Thalmair, W. Bolz, and H. Wachtel, "Immediate loading of fixed provisional prostheses using four implants for the rehabilitation of the edentulous arch: a prospective clinical study," The International Journal of Oral \& Maxillofacial Implants, vol. 25, no. 5, pp. 1011-1018, 2010.

[2] M. Chiapasco, "Early and immediate restoration and loading of implants in completely edentulous patients," The International Journal of Oral \& Maxillofacial Implants, vol. 19, pp. 76-91, 2004.

[3] M. Del Fabbro, T. Testori, L. Francetti, S. Taschieri, and R. Weinstein, "Systematic review of survival rates for immediately loaded dental implants," International Journal of Periodontics and Restorative Dentistry, vol. 26, no. 3, pp. 249-263, 2006.

[4] R. Crespi, P. Capparè, G. Gastaldi, and E. F. E. Gherlone, "Immediate occlusal loading of full-arch rehabilitations: screwretained versus cement-retained prosthesis. An 8-year clinical evaluation," The International journal of oral \& maxillofacial implants, vol. 29, no. 6, pp. 1406-1411, 2014.

[5] P. Maló, B. Rangert, and M. Nobre, “'All-on-four' immediatefunction concept with brånemark system implants for completely edentulous mandibles: a retrospective clinical study," Clinical Implant Dentistry and Related Research, vol. 5, supplement 1, pp. 2-9, 2003.

[6] P. Maló, B. Rangert, and M. Nobre, "All-on-4 immediatefunction concept with Brånemark System implants for completely edentulous maxillae: a 1-year retrospective clinical study," Clinical Implant Dentistry and Related Research, vol. 7, supplement 1, pp. S88-S94, 2005. 
[7] J. Duyck, H. Van Oosterwyck, J. V. Sloten, M. De Cooman, R. Puers, and I. Naert, "Magnitude and distribution of occlusal forces on oral implants supporting fixed prostheses: An in vivo study," Clinical Oral Implants Research, vol. 11, no. 5, pp. 465475, 2000.

[8] G. Sannino, "All-on-4 concept: a 3-dimensional finite element analysis," Journal of Oral Implantology, vol. 41, no. 2, pp. 163171, 2015.

[9] E. L. Agliardi, A. Pozzi, C. F. J. Stappert, R. Benzi, D. Romeo, and E. Gherlone, "Immediate fixed rehabilitation of the edentulous maxilla: A prospective clinical and radiological study after 3 years of loading," Clinical Implant Dentistry and Related Research, vol. 16, no. 2, pp. 292-302, 2014.

[10] E. Gherlone, P. Capparé, R. Vinci, F. Ferrini, G. Gastaldi, and R. Crespi, "Conventional versus digital impressions for "Allon-Four" restorations," The International Journal of Oral \& Maxillofacial Implants, vol. 31, no. 2, pp. 324-330, 2016.

[11] L. Krekmanov, M. Kahn, B. Rangert, and H. Lindström, "Tilting of posterior mandibular and maxillary implants for improved prosthesis support," The International Journal of Oral \& Maxillofacial Implants, vol. 15, no. 3, pp. 405-414, 2000.

[12] R. Calandriello and M. Tomatis, "Simplified treatment of the atrophic posterior maxilla via immediate/early function and tilted implants: a prospective 1-year clinical study," Clinical Implant Dentistry and Related Research, vol. 7, no. 1, pp. S1-S12, 2005.

[13] C. Kunavisarut, L. A. Lang, B. R. Stoner, and D. A. Felton, "Finite element analysis on dental implant-supported prostheses without passive fit," Journal of Prosthodontics, vol. 11, no. 1, pp. 30-40, 2002.

[14] G. Sannino, P. Bollero, A. Barlattani, and E. Gherlone, "A retrospective 2-year clinical study of immediate prosthetic rehabilitation of edentulous jaws with four implants and prefabricated bars," Journal of Prosthodontics, vol. 26, no. 5, pp. 387394, 2017.

[15] M. B. Hurzeler, A. Kirsch, K. L. Ackermann, and C. R. Quinones, "Reconstruction of the severely resorbed maxilla with dental implants in the augmented maxillary sinus: a 5year clinical investigation," The International Journal of Oral \& Maxillofacial Implants, vol. 11, pp. 466-475, 1996.

[16] J. I. Smedberg, E. Lothigius, I. Bodin, A. Frykholm, and K. Nilner, "A clinical and radiological two-year follow-up study of maxillary overdentures on osseointegrated implants," Clinical Oral Implants Research, vol. 4, no. 1, pp. 39-46, 1993.

[17] Y. Fortin, R. M. Sullivan, and B. R. Rangert, “The Marius implant bridge: Surgical and prosthetic rehabilitation for the completely edentulous upper jaw with moderate to severe resorption: A 5year retrospective clinical study," Clinical Implant Dentistry and Related Research, vol. 4, no. 2, pp. 69-77, 2002.

[18] G. Sannino and A. Barlattani, "Straight versus angulated abutments on tilted implants in immediate fixed rehabilitation of the edentulous mandible: A 3-year retrospective comparative study," International Journal of Prosthodontics, vol. 29, no. 3, pp. 219-226, 2016.

[19] A. O. Spazzin, M. B. F. dos Santos, L. C. Sobrinho, R. L. X. Consani, and M. F. Mesquita, "Effects of horizontal misfit and bar framework material on the stress distribution of an overdenture-retaining bar system: A 3D finite element analysis," Journal of Prosthodontics, vol. 20, no. 7, pp. 517-522, 2011.
[20] S.-R. Ha, S.-H. Kim, S.-I. Song, S.-T. Hong, and G.-Y. Kim, "Implant-supported overdenture with prefabricated bar attachment system in mandibular edentulous patient," The Journal of Advanced Prosthodontics, vol. 4, no. 4, pp. 254-258, 2012.

[21] A. C. M. Melo, M. C. De Freitas, S. R. Bernardes, I. A. De Mattias Sartori, A. P. F. Bassi, and G. Thomé, "A prospective follow-up study of 44 mandibular immediately loaded implants using resonance frequency analysis: Preliminary 1-Year results," Implant Dentistry, vol. 18, no. 6, pp. 530-538, 2009.

[22] M. A. Abd El-Dayem, A. S. Assad, M. E. Eldin Sanad, and S. A. A.-H. Mahmoud Mogahed, "Comparison of prefabricated and custom-made bars used for implant-retained mandibular complete overdentures," Implant Dentistry, vol. 18, no. 6, pp. 501-511, 2009. 


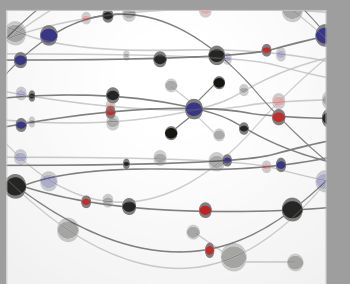

The Scientific World Journal
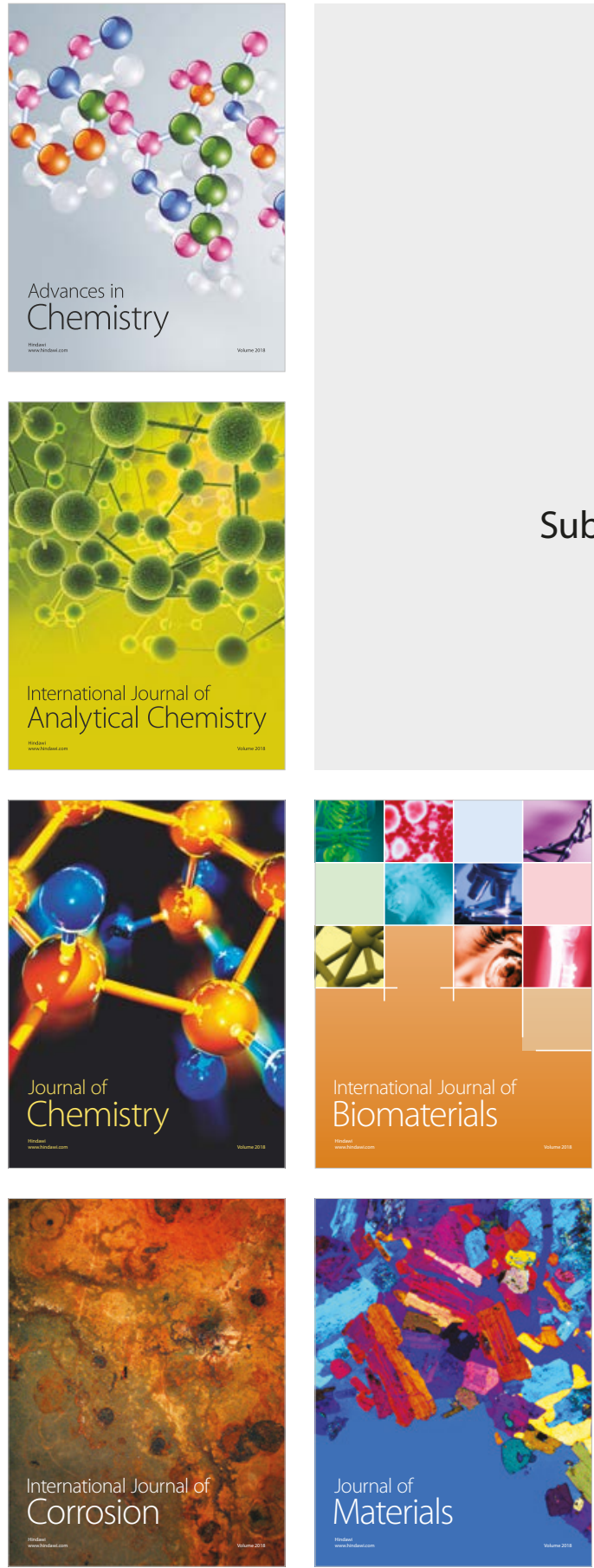

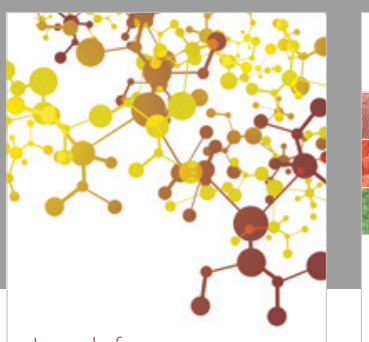

Journal of

Applied Chemistry
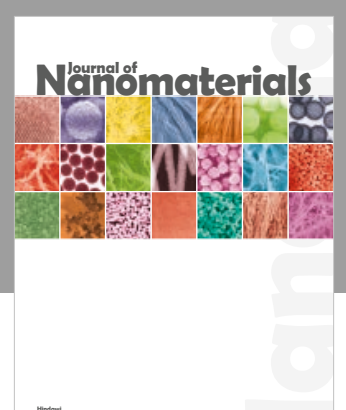

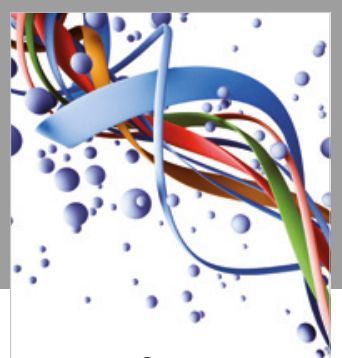

Scientifica

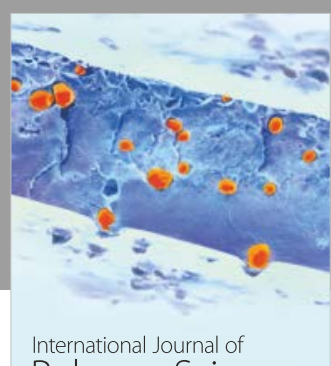

Polymer Science

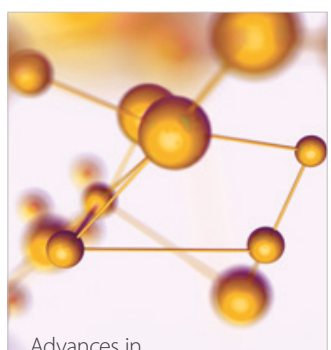

Physical Chemistry
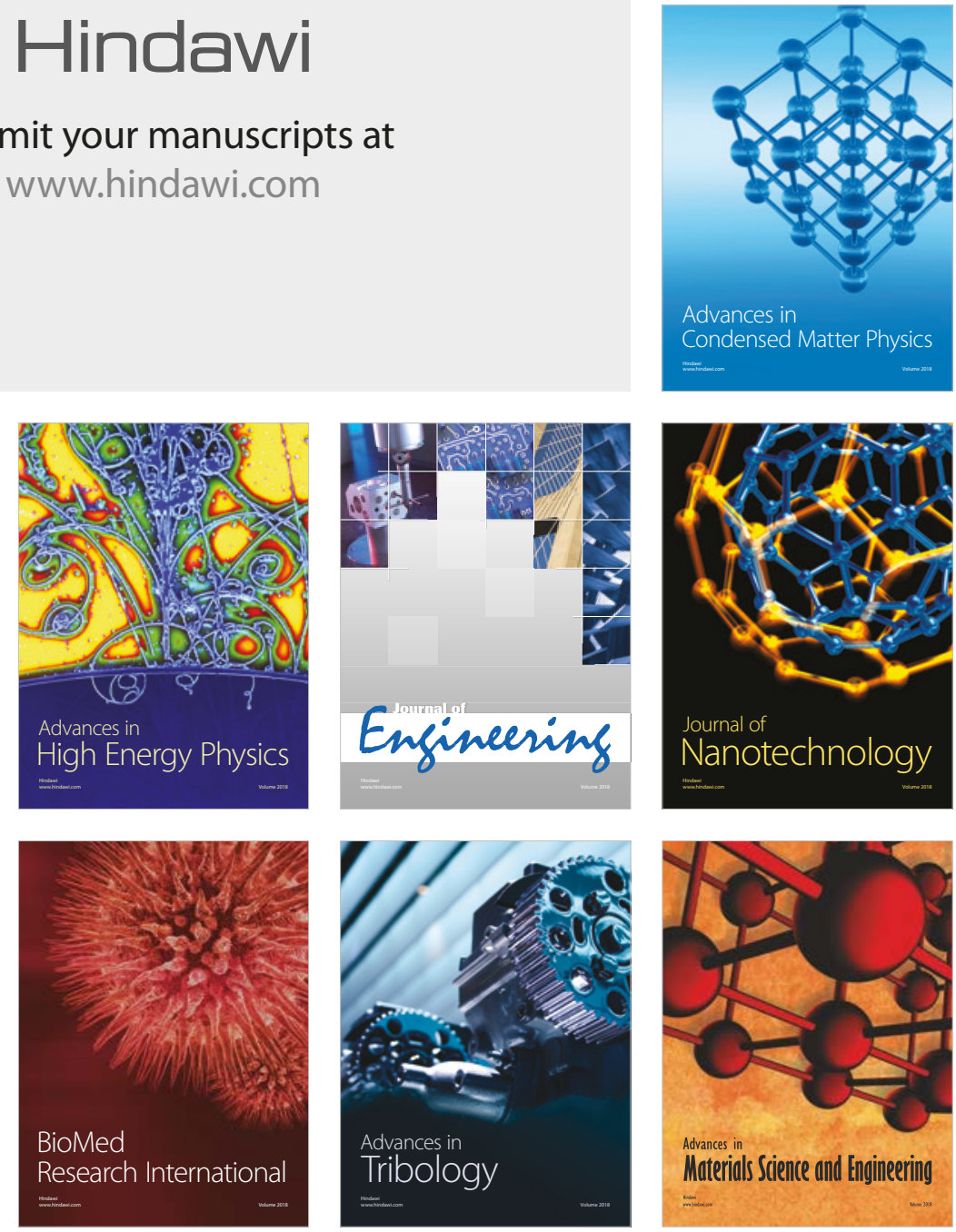